\title{
RESEARCH HIGHLIGHT Water quality check: macrophages setting the standards
}

\author{
Camille Blériot ${ }^{1}$, Zhaoyuan Liu $^{2}$ and Florent Ginhoux (D) $2,3,4$ \\ Cell Research (2021) 31:3-4; https://doi.org/10.1038/s41422-020-00429-x
}

\begin{abstract}
In a recent study published in Cell, Chikina et al. uncovered a new mechanism by which colonic macrophages perform quality check of fluids absorbed by colonic epithelial cells. This critical function allows the maintenance of barrier integrity by avoiding intoxication with fungal toxins and the resulting life-threatening risk of colonocyte apoptosis.

Macrophages are innate immune cells well-known for their phagocytic functions of hazardous intruders but they are also involved in the maintenance of healthy tissue homeostasis. In many organs, macrophages seed tissues during embryonic development thus developing a very tight functional relationship with their niche of residence. ${ }^{\top}$ On the contrary, in the gut, besides of a minor population of long-lived embryonic macrophages essentially located in the muscular layer, ${ }^{2,3}$ the majority of colonresident macrophages derive from adult monocytes and are continuously replenished by circulating cells during adulthood. ${ }^{4}$ This singularity is supposed to result from their specific and challenging exposure to microbiota, rendering this niche particularly dynamic and thus not adapted to a permanent population of embryonic long-lived macrophages.
\end{abstract}

A recent study published in Cell ${ }^{5}$ provides new and unexpected insights into the functional roles of macrophages residing in the colonic mucosa. Firstly, using the CD64 ${ }^{D T R}$ mouse model to specifically deplete tissue-resident macrophages, ${ }^{6}$ the authors observed that colonic barrier integrity was severely impaired, with colonocytes undergoing massive apoptosis upon macrophage depletion. To investigate the underlying mechanisms of action, they adopted a powerful confocal microscopy-based approach to closely look at colonic macrophages in situ and identified a previously undescribed feature of distal colon macrophages, "balloon-like" protrusions (BLPS) inserted in the epithelial layer but not in contact with the colonic lumen which is separated from colonocytes by a thick layer of mucus.

The authors next investigated the potential functions of these BLPs. It has been previously demonstrated that colonic macrophages, defined by a high expression of the fractalkine receptor, CX3CR1, controlled immunity to intestinal fungi. ${ }^{7}$ In line with this, the present study showed that anti-fungal (fluconazole or amphotericin B) treatment decreased the number of BLPs but did not affect the number of total macrophages. More interestingly, they showed that these antifungal but not antibiotic treatments prevented the massive apoptosis of epithelial cells observed in the absence of macrophages, demonstrating that fungi are responsible for epithelial cell death in macrophage-depleted mice, or in other words BLP macrophages (BLP-Mac) were needed to protect epithelial cells from a massive fungal-induced death. Furthermore, the authors observed that BLP-Mac response was strikingly independent of the recognition of fungal cell wall glucans by the classical receptors Dectin 1 and Dectin $2,{ }^{7}$ arguing for an original mechanism at play in this context. The authors reported that BLP-Mac indirectly sampled fungal toxins through the fluids absorbed by colonocytes. By modulating osmotic pressure in the colon, authors observed that BLPs were dynamic structures, being increased when mice were treated with a hypotonic solution or reduced when mice were treated with laxatives. Finally, by using purified fungal toxins, the authors observed that colonocytes could sense them and stop absorbing contaminated fluids, a capacity that was lost in CD64 DTR macrophage-depleted mice. Therefore, by avidly absorbing fungal toxin-enriched fluids, BLP-Mac act as natural sponges, modulating sensing capacity of colonocytes and preventing them from death by fungal toxin absorption.

Overall, this study combined several powerful and state of the art technologies to uncover a new function of macrophages. It is an additional proof of the astonishing versatility of functions of these innate immune cells. In the specific gut context, intestinal macrophages have been the focus of many studies, e.g., the presence of luminal extensions of $\mathrm{CX} 3 \mathrm{CR} 1^{+}$cells in the small intestine has been reported almost twenty years ago. ${ }^{8}$ Noteworthy, small intestine and colon are two distinct niches with notably a far thicker layer of mucus in the colon. Therefore, structure and functions of macrophage protrusions, colon BLPs vs small intestine luminal extensions, are fundamentally different. Even if direct sensing of the colon lumen is restricted due to tissue organization, results from this study show that colonic macrophages have developed BLPs as unique and specific adaptations to their local environment. Considering that it has been demonstrated that mucus absorption by myeloid cells in the small intestine leads to the generation of immunoregulatory signals, ${ }^{9}$ it would be relevant to investigate whether a similar phenomenon occurs in colon and how these BLPs are involved. Moreover, while the present study has essentially assessed the quantity of BLPs depending on fluctuating conditions in the tissue, further studies should clarify their stability notably to understand if they can expand or retract, or even disappear in response to various stimuli, with relevance for pathologies. Another question is also related to the kinetics of their appearance considering that colonic macrophages are mostly derived from short-lived monocytes, BLPs should be formed quite quickly after engraftment of naive monocytes and differentiation in resident macrophages. Identifying signals required for this process will be definitely of interest and should provide tools to modulate abundance of BLPs, a strategy that could be useful notably for the treatment of intestinal specific pathologies such as inflammatory bowel disease or colon cancer.

\footnotetext{
${ }^{1}$ Inserm U1015, Gustave Roussy, Villejuif 94800, France; ${ }^{2}$ Shanghai Institute of Immunology, Shanghai Jiao Tong University School of Medicine, Shanghai 200025, China;

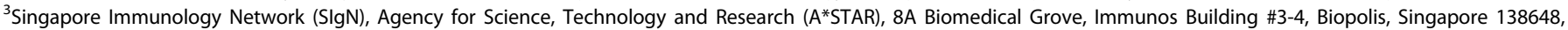
Singapore and ${ }^{4}$ Translational Immunology Institute, SingHealth Duke-NUS Academic Medical Centre, Singapore 169856, Singapore

Correspondence: Florent Ginhoux (Florent_Ginhoux@immunol.a-star.edu.sg)
} 
Although being quite complete, this study also opens several questions that will need to be tackled in future studies. First, the authors have observed that BLPs were filled with epithelial cell membranes at steady state, suggesting that colonic macrophages could actively phagocyte epithelial cells continuously. Therefore, the relative importance of the homeostatic recycling of colonocytes, independent of fungal toxin absorption, remains to be determined. In addition, a limitation of the study comes from the depletion model used. Indeed, CD64 expression is assumed to be restricted to macrophages, but a CD64-based depletion will affect virtually all macrophages in the organism. At the colon level, it means that not only epithelial-associated BLP-Mac will be removed but also all the non-BLP-Mac. Furthermore, macrophages from other tissues will also be impacted. A direct role of distant cells in the phenomenon reported in the present study can presumably be ruled out, but it could have implications in general physiology.

An exciting finding of this study is the role of CD74 in BLP formation in response to toxin-enriched fluid absorption by colonic macrophages. Indeed, the authors observed a defect in BLP formation in $C D 74^{K O}$ mice, which they hypothesize being due to the role of CD74 in expanding the endolysosomal compartments enclosed in BLPs. While plausible, it is relevant to note that CD74 has pleiotropic roles, being notably able to act as a transcription factor. ${ }^{10}$ Thus, an additional indirect role of CD74 through transcription regulation of yet unidentified factors cannot be ruled out and deserves further studies.
The authors also provided a transcriptional analysis of the BLPMac at the single cell level and notably found an increased expression of the gene Itgax encoding CD11 c in this subset. A recent study has identified a subset of $\mathrm{CD}_{11 \mathrm{c}^{+}}$macrophages associated with ductal epithelial layer in mammary glands. ${ }^{11}$ These cells have been shown to continuously sense ductal epithelium and maintain their integrity at steady state while being involved in remodeling during lactation or potentially tumorigenesis. Obviously, mammary glands and colon are two distinct niches with their own specificities but it is amazing to see that their resident macrophage populations could have evolved separately to assume similar functions. Once again, it highlights the tremendous adaptability of macrophages and argues for the need of further studies to better apprehend it, with the aim to target them in many pathologies.

\section{REFERENCES}

1. Bleriot, C. et al. Immunity 52, 957-970 (2020).

2. Shaw, T. N. et al. J. Exp. Med. 215, 1507-1518 (2018).

3. De Schepper, S. et al. Cell 175, 400-415 (2018).

4. Bain, C. C. et al. Nat. Immunol. 15, 929-937 (2014).

5. Chikina, A. S. et al. Cell https://doi.org/10.1016/j.cell.2020.08.048 (2020).

6. Baranska, A. et al. J. Exp. Med. 215, 1115-1133 (2018).

7. Leonardi, I. et al. Science 359, 232-236 (2018).

8. Rescigno, M. et al. Nat. Immunol. 2, 361-367 (2001).

9. Shan, M. et al. Science 342, 447-453 (2013).

10. Gil-Yarom, N. et al. Proc. Natl. Acad. Sci. USA 114, 562-567 (2017).

11. Dawson, C. A. et al. Nat. Cell Biol. 22, 546-558 (2020). 